\author{
ATUAÇÃO DO FISIOTERAPEUTA NO TRATAMEN- \\ TO DA DOR GÊNITO PÉLVICA/PENETRAÇÃO \\ COM FOCO NA ABORDAGEM DA TERAPIA MANU-
}

AL EM MULHERES NA MENACME

\title{
PHYSIOTHERAPEUTIC ACTIVITY IN THE TRE- ATMENT OF PENETRAL GENE PAIN / PENETRA- TION WITH A FOCUS ON THE MANUAL THERAPY APPROACH IN WOMEN AT MENACME
}

Resumo: $\mathrm{O}$ transtorno da dor gê- durante a tentativa de penetranito-pélvica/penetração (DGPP) ção vaginal. A função sexual engloba o vaginismo, dispareu- adequada é considerada um fania e a vulvodinia, onde se apre- tor importante para qualidade sentam os seguintes sintomas: de vida, tendo reconhecimento Presença de medo ou ansiedade da OMS (Organização Mundial relacionado à dor vulvovaginal da Saúde) quanto à presença da ou pélvica em antecipação, du- disfunção sexual como problema rante ou como resultado de pene- de saúde pública, recomendando tração vaginal, além da presença de tensão ou endurecimento dos sua investigação por causar importantes alterações na qualidade músculos do assoalho pélvico de vida e no relacionamento com

1 Bacharel em Fisioterapia - Universidade Celso Lisboa. Pós Graduando em Fisioterapia Pélvica (Uroginecologia funcional) - Instituto Inspirar

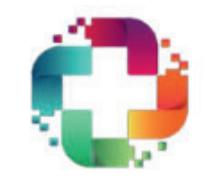


ISSN: $2763-5724$

Vol. 01 - n 05 - ano 2021

Editora Acadêmica Periodicojs

seu parceiro. Identifica-se que a atuação do fisioterapeuta se torna relevante no processo de identificação e reabilitação das dores gênito-pélvicas, justificando a importância de estudos nesse tema. Com o objetivo de analisar a atuação do fisioterapeuta no tratamento da dor gênito pélvica/ penetração com foco na abordagem da terapia manual em mulheres na menacme, foi realizada uma revisão narrativa da literatura onde foram utilizados 17 artigos como base para a pesquisa e coleta de dados. Durante as análises dos artigos definiu-se que as DSFs tendem a aparecer na fase fértil feminina, causando dores e desconfortos. Nas revisões abordadas nesse estudo, analisou-se que DGPP é uma síndrome complexa na qual a sensação e intensidade da dor são sentidas em diversos locais. Com isto o diagnóstico se torna difícil levando em consideração a vergonha e/ ou bloqueio da mulher em relatar uma queixa sexual. A partir de uma avaliação fisioterapêutica, a fim de verificar o tônus e a função da musculatura do assoalho pélvico, a existência e localização de pontos dolorosos, bem como a compreensão de contrações e repousos voluntários dessas musculaturas, é traçado um planejamento terapêutico com uso de intervenções específicas que constam em técnicas de auto-relaxamento e controle da ansiedade, melhorando a consciência corporal. Conclui-se que há eficácia em explorar o autoconhecimento para melhora da qualidade de vida das mulheres que sofrem deste problema, proporcionando resultados positivos em um curto período, contribuindo então para a melhorar da função sexual de mulheres com DSFs. 
Palavras-chaves: Fisioterapia; changes in quality of life and in Dor Gênito Pélvica; Terapia Ma- the relationship with your partnual; Disfunção Sexual Feminina.

ner. It is identified that the role of the physiotherapist becomes relevant in the process of identifi-

Abstract: Genital-pelvic pain / penetration disorder (DGPP) includes vaginismus, dyspareunia and vulvodynia, where the following symptoms are present: Presence of fear or anxiety related to vulvovaginal or pelvic pain in anticipation, during or as a result of vaginal penetration, in addition to the presence of tension or hardening of the pelvic floor muscles during the attempt of vaginal penetration. Adequate sexual function is considered an important factor for quality of life, being recognized by WHO (World Health Organization) regarding the presence of sexual dysfunction as a public health problem, recommending its investigation for causing important cation and rehabilitation of genito-pelvic pain, justifying the importance of studies on this topic. In order to analyze the role of the physiotherapist in the treatment of pelvic genital pain / penetration with a focus on the approach of manual therapy in women at menacme, a narrative review of the literature was carried out, where 17 articles were used as a basis for research and data collection. During the analysis of the articles, it was defined that DSFs tend to appear in the female fertile phase, causing pain and discomfort. In the reviews covered in this study, it was analyzed that DGPP is a complex syndrome in which the sensation and intensity of pain are felt in diffe-

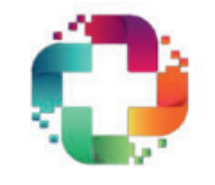


ISSN: $2763-5724$

Vol. 01 - n 05 - ano 2021

Editora Acadêmica Periodicojs

rent places. With this the diagno-

sis becomes difficult taking into account the shame and / or blocking of the woman in reporting a sexual complaint. Through a physical therapy assessment, in order to check the tone and function of the pelvic floor muscles, the existence and location of tender points, as well as the understanding of voluntary contractions and rests of these muscles, a therapeutic planning with the use of interventions is outlined. specific measures in self-relaxation and anxiety control techniques, improving body awareness. It is concluded that there is effectiveness in exploring self-knowledge to improve the quality of life of women who suffer from this problem, providing positive results in a short period, thus contributing to the improvement of the sexual function of women with DSFs (Female Sexual Dysfunc- tions).

Keywords: Physiotherapy; Pelvic Genital Pain; Manual Therapy; Female Sexual Dysfunction.

\section{INTRODUÇÃO}

Com o objetivo de analisar a atuação do fisioterapeuta no tratamento da dor gênito pélvica/ penetração com foco na abordagem da terapia manual em mulheres na menacme, o presente artigo apresenta uma revisão narrativa da literatura onde foram utilizados 17 artigos como base para a pesquisa e coleta de dados.

O corpo da mulher passa por diversas fases no decorrer da vida, as DSFs tendem a aparecer na fase fértil feminina e podem se caracterizar como dificuldade persistente ou recorrente de alcançar a penetração vaginal durante o coito, ou dor vulvova-

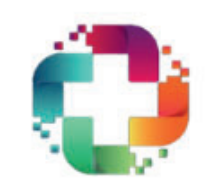


ISSN: 2763-5724

Vol. 01 - n 05 - ano 2021

Editora Acadêmica Periodicojs

ginal ou pélvica durante relação sexual ou tentativa de penetração vaginal (APA, 2013; LAHAIE, 2015). As DSFs acometem cerca de $67,9 \%$ das mulheres no mundo, estimando-se a presença de 30 a $50 \%$ das mulheres norte-americanas, em mais de $50 \%$ das asiáticas e em $30 \%$ das brasileiras (WOLPE et al., 2015).

A função sexual adequada é considerada um fator importante para qualidade de vida, tendo reconhecimento da OMS (Organização Mundial da Saúde) quanto à presença da disfunção sexual como problema de saúde pública, recomendando sua investigação por causar importantes alterações na qualidade de vida e no relacionamento com seu parceiro. Identifica-se que a atuação do fisioterapeuta se torna relevante no processo de identificação e reabilitação das dores gênito-pélvicas, justificando a im- portância de estudos nesse tema.

Durante as análises dos artigos definiu-se que as DSFs tendem a aparecer na fase fértil feminina, causando dores e desconfortos. Nas revisões abordadas nesse estudo, analisou-se que DGPP é uma síndrome complexa na qual a sensação e intensidade da dor são sentidas em diversos locais. Com isto o diagnóstico se torna difícil levando em consideração a vergonha e/ou bloqueio da mulher em relatar uma queixa sexual. A partir de uma avaliação fisioterapêutica, a fim de verificar o tônus e a função da musculatura do assoalho pélvico, a existência e localização de pontos dolorosos, bem como a compreensão de contrações e repousos voluntários dessas musculaturas, é traçado um planejamento terapêutico com uso de intervenções específicas que constam em técnicas de auto-relaxamento e con-

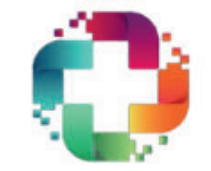


ISSN: $2763-5724$

Vol. 01 - n 05 - ano 2021

Editora Acadêmica Periodicojs

trole da ansiedade, melhorando a consciência corporal.

As disfunções sexuais femininas (DSFs) são consideradas, pela Organização Mundial da Saúde (OMS), um problema de saúde pública, uma vez que podem afetar mulheres em períodos de curto, médio e longo prazo de suas vidas, tendo grande impacto também na vivência social, psicológica, doméstica, ocupacional e física das pacientes e de seus parceiros (WOLPE, 2015).

Os resultados do artigo evidenciam que há eficácia em explorar o autoconhecimento para melhora da qualidade de vida das mulheres que sofrem deste problema, proporcionando resultados positivos em um curto período, contribuindo então para a melhorar da função sexual de mulheres com DSFs. A contribuição do estudo para o campo da Saúde está na necessidade de se pensar políticas públicas que ofereçam às mulheres tratamento adequado para o tratamento das disfunções sexuais femininas.

\section{MENACME}

A mulher tem diferentes tipos de ciclo reprodutor desde a infância até a idade adulta. A menarca marca o fim da infância (puberdade) e a menacme é o período fértil feminino que dura da primeira menstruação até a última, denominada menopausa, que se dá depois de um ano de ausência da menstruação. O climatério é o período de transição do período fértil para o período não reprodutivo causado pela baixa produção hormonal e da função ovariana e é divido em perimenopausa (diminuição da fertilidade) até a menopausa e a pós menopausa (todo tempo depois da última menstruação) (BASILIO 
ISSN: 2763-5724

Vol. 01 - n 05 - ano 2021

Editora Acadêmica Periodicojs

et al., 2016).

Helen também apontou o desejo como parte da fase prévia cere-

DISFUNÇÃO SEXUAL FE- bral e propôs um modelo divido MININA

em três fases: desejo, excitação e orgasmo (RIBEIRO et al., 2013).

É parte integrante e imJá em 2005, Basson fez portante da vida do ser humano uma nova análise. Neste modelo a satisfação na vida sexual, pois a mulher pode iniciar o ato com faz parte do bem-estar próprio e nas relações afetivas. A sexualidade é influenciada de diferentes formas, pois leva em conta a personalidade, fatores biológicos, o ciclo da vida, as experiências sexuais prévias e o psicológico (VIEIRA et al., 2016)

A resposta sexual feminina e masculina foi descrita à primeira vez por Masters e Jonhson em 1966, que a caracterizou em quatro fases: excitação, plateau, orgasmo e resolução. Tempos depois, Helen Kaplan em 1979, apontou diferenças entre os sexos, pois o homem tem ou sem consciência do desejo sexual, e isso ocorre porque é receptiva e responsiva ao estímulo gerado que resulta em excitação subjetiva com a resposta física, ou porque tem excitação espontânea que desencadeia a consciência do desejo sexual que a leva a excitação e mais desejo. Sua resposta pode levar ou não ao alívio orgástico, mas através dessa análise fica claro que a mulher mesmo não atingindo o orgasmo na relação sexual, pode se sentir totalmente satisfeita (LARA et al., 2008).

Atualmente, de acordo período refratário e mulher não, com o Manual Diagnóstico e Es-

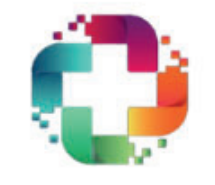


ISSN: $2763-5724$

Vol. 01 - n 05 - ano 2021

Editora Acadêmica Periodicojs

tatístico de Transtornos Mentais (DSM-V), o transtorno de dor gênito-pélvica/penetração (DGPP) engloba a dispareunia, o vaginismo e a vulvodinia, representando uma única disfunção (FEBRASGO, 2017).

O Transtorno de Dor Gênito-Pélvica/Penetração baseia-se na dificuldade persistente ou recorrente de alcançar a penetração vaginal durante o coito, dor vulvovaginal ou pélvica durante relação sexual ou tentativa de penetração vaginal (BRASIL e $A B D O, 2016)$.

É uma síndrome bem complexa, onde a sensação e intensidade da dor são sentidas em diversos locais, ocorrendo distúrbios musculoesqueléticos e miofasciais, o que contribui significativamente para a disfunção sexual feminina (BERGHMANS, 2018).

Caracteriza-se também pela presença de medo ou ansiedade relacionado à dor vulvovaginal ou pélvica em antecipação, durante ou como resultado de penetração vaginal, além da presença de tensão ou endurecimento dos músculos do assoalho pélvico durante a tentativa de penetração vaginal (PAZMANY et al., 2017).

O sofrimento do indivíduo e a exclusão de transtornos mentais, violência sexual pelo parceiro ou outros fatores estressores que possam resultar em dor sexual também deve ser considerada para estabelecimento do diagnóstico (APA, 2013; LAHAIE et al., 2015).

A dispareunia, classificada como distúrbio gênito pélvico de dor/penetração, é caracterizada pela queixa de dor persistente ou recorrente, podendo ocorrer antes, durante ou após a relação sexual, sendo comum 
em mulheres em idade reprodutiva (GHADERI et al., 2019).

Pode ser classificada como superficial ou profunda e é definida como a presença de dor genital durante a relação sexual, podendo estar associada a fatores físicos e/ou psicológicos (MORRIS et al., 2006).

A dispareunia superficial refere-se à dor percebida em região vulvovestibular no início da penetração, ou com o movimento do pênis dentro da vagina durante a relação sexual, e pode ter múltiplas etiologias, principalmente relacionadas com patologias genitais ou pélvicas como: atrofia genital na pós-menopausa, ressecamento vaginal e lubrificação vaginal inadequada, infecção do trato urinário, prolapso, líquen, entre outros (BINIK, 2010).

Já a dispareunia de profundidade, na qual a dor se ma- nifesta no fundo vaginal e hipogástrio, está frequentemente associada a um padrão de dor que se reproduz em outras situações fora do ato sexual e dor pélvica crônica propriamente dita, tendo causa predominantemente orgânica. Pode ser persistente, quando a dor ocorre em todas as relações, ou condicional, quando a dor se apresenta em algumas posições, tipos de estimulações ou relacionada a um parceiro específico (HEIM et al., 2001; MORRIS et al., 2006).

O vaginismo caracteriza-se pela dificuldade na penetração vaginal associada à dor, ao medo e à contração da musculatura do assoalho pélvico no momento da relação sexual. O vaginismo pode ser primário quando a mulher nunca conseguiu ter um coito ou secundário, o qual em geral deve-se ao surgimento de dispareunia (LAHAIE et al.,

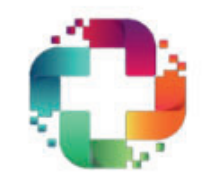


ISSN: 2763-5724

Vol. 01 - n 05 - ano 2021

Editora Acadêmica Periodicojs

2015).

terminações nervosas na vulva e

As mulheres com vagivagina (STRAUB, 2007).

nismo normalmente apresentam

A terapia manual é basdesejo, excitação e orgasmo com tante eficaz no tratamento das outros tipos de relação onde não DSFs causada pela tensão dos haja penetração. Apresentam lubrificação vaginal e são anorgásmicas, porém incapazes de ter o coito (APA, 2013).

A vuldodínia é caracterizada pelo desconforto vulvar, queimação na vulva e vestíbulo vaginal. Pode ser classificada em espontânea ou provocada (por contato, relação sexual) e em generalizada ou localizada (vestibulodínia, clitorodínia, hemivulvodínia) (BORNSTEIN et al., 2016).

Entre os fatores hormonais, o hipoestrogenismo associado ao estado menopausa, e que pode estar associado à síndrome geniturinária da menopausa, tem importante papel já que pode levar ao aumento de densidade de músculos do assoalho pélvico, resultando no alívio da dor a longo prazo, na melhora da função sexual e consequentemente, melhor qualidade de vida (SILVA et al., 2017). O problema é desenvolver uma política pública que possa levar alívio às mulheres, seja pelo tabu que envolve a questão relacionada à sexualidade, seja por ausência de priorização do poder público quanto às DSFs, afinal, e mulher ainda não alcançou o seu lugar de respeito na sociedade machista em que vivemos, cf. demonstraram Mendonça e Berto (2021).

As manobras realizadas ajudam na diminuição das aderências decorrentes de intercorrências ginecológicas que podem 
ISSN: 2763-5724

Vol. 01 - n 05 - ano 2021

Editora Acadêmica Periodicojs

provocar a dispareunia e disfunção no orgasmo na mulher, pois trabalha no relaxamento da musculatura pélvica e na vascularização local (WOLPE et al., 2015).

\section{FISIOTERAPIA NA SAÚDE} DA MULHER

O fisioterapeuta especializado na saúde da mulher tem papel de aconselhar, identificar e tratar a disfunção do assoalho pélvico, atuando na prevenção e no tratamento da mesma, sendo definida como função anormal do assoalho pélvico podendo ter impactos prejudiciais e significativos na qualidade de vida da mulher (LAWSON e SACKS, 2018).

A Fisioterapia na saúde da mulher utiliza de métodos e técnicas voltados para o corpo feminino como um todo, uma vez que, mulheres com problemas de constipação, retenção urinária, incontinência urinária, incontinência fecal, prolapsos genitais ("bexiga caída"), além dos mais diversos tipos de disfunção sexual, podem recorrer a essa especialidade da fisioterapia para o alívio e, por vezes, à solução total destes inconvenientes (LATORRE, 2010).

Com o aumento do empoderamento feminino, vê-se um maior interesse da população e da comunidade científica sobre as disfunções sexuais femininas, visto que o tema está totalmente atrelado à saúde da mulher, sendo assim, a atuação do fisioterapeuta se torna relevante no processo de identificação e reabilitação das dores gênito-pélvicas.

Dado este fato propõe-se a realização de novos estudos envolvendo o tema, objetivando estruturar e fundamentar as ações ligadas ao processo reabilitativo, garantindo assim uma 
ISSN: $2763-5724$

Vol. 01 - n 05 - ano 2021

Editora Acadêmica Periodicojs

atuação mais eficiente.

guns artigos anteriores a esse

ano foram utilizados por se tra-

METODOLOGIA, ANÁLISE tarem de estudos pertinentes ao

E DISCUSSÃO DO MATEtema. Na coleta de dados seleRIAL

cionamos todos os artigos com

informações sobre as DDGPs e

Para esse estudo, foi a terapia manual como método realizada uma revisão narrativa de tratamento. Como critério de da literatura no período de 18 de exclusão, descartamos artigos soMarço de 2020 a 20 de Junho de bre disfunções masculinas, sobre 2020, a qual foram selecionados tratamento focado em eletroestipor busca eletrônica em bases de mulação ou qualquer outro tipo dados pelas plataformas PEDro, de aparelho e que continham inPubMed e SciELO. Os descri- continência urinária associada às tores pesquisados foram: Dis- disfunções. Após isso foram orfunção sexual feminina, terapia ganizados em formato de tabela manual, menacme, dor gênito para melhor comparação de suas pélvica, fisioterapia, dispareunia, informações. Para a presente revaginismo, vulvodinia (female visão, foram utilizados 17 artigos sexual dysfunction, manual thecomo base para a pesquisa e corapy, pelvic genital pain, physioleta de dados.

therapy, dyspareunia, vaginismus, vulvodynia).

A busca foi limitada para artigos publicados entre os anos de 2015 - 2020. Porém, al-

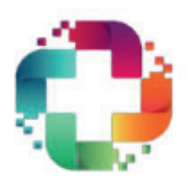


ISSN: 2763-5724

Vol. 01 - n 05 - ano 2021

Editora Acadêmica Periodicojs

QUADRO 1 - METODOLOGIA E ANÁLISE DE DADOS SOBRE DISFUNÇÕES SEXUAIS FEMININAS:

ABORDAGENS, TÉCNICAS E TRATAMENTOS

\begin{tabular}{|c|c|c|c|}
\hline ARTIGO & AUTORES / ANO & METODOLOGIA & RESULTADOS \\
\hline $\begin{array}{l}\text { Abordagem das } \\
\text { disfunções sexuais } \\
\text { femininas }\end{array}$ & LARA et al., 2008 & $\begin{array}{l}\text { Pesquisa explicativa, } \\
\text { mostrando como } \\
\text { utilizar o método Pilset }\end{array}$ & $\begin{array}{l}\text { Impacto positivo do método } \\
\text { Pilset na função sexual }\end{array}$ \\
\hline $\begin{array}{l}\text { Fisioterapia na } \\
\text { Saúde da Mulher }\end{array}$ & LATORRE, 2010 & $\begin{array}{l}\text { Conceito sobre a } \\
\text { fisioterapia na saúde } \\
\text { da mulher }\end{array}$ & $\begin{array}{l}\text { Os recursos da fisioterapia } \\
\text { servem para diversos } \\
\text { problemas voltados a saúde } \\
\text { da mulher e sua prevenção }\end{array}$ \\
\hline $\begin{array}{c}\text { Diagnostic and } \\
\text { Statistical Manual of } \\
\text { Mental Disorders }\end{array}$ & $\begin{array}{c}\text { AMERICAN } \\
\text { PSYCHIATRIC } \\
\text { ASSOCIATION, } 2013\end{array}$ & $\begin{array}{l}\text { Critérios concisos e } \\
\text { explícitos, com } \\
\text { objetivo de facilitar } \\
\text { avaliação das } \\
\text { apresentações dos } \\
\text { sintomas em uma } \\
\text { variedade de } \\
\text { ambientes clínicos }\end{array}$ & $\begin{array}{c}\text { Classificação das disfunções } \\
\text { sexuais contendo } \\
\text { especificadores como } \\
\text { subtipos e gravidade atual }\end{array}$ \\
\hline $\begin{array}{l}\text { Disfunção sexual } \\
\text { feminina em idade } \\
\text { reprodutiva - } \\
\text { Prevalência e fatores } \\
\text { associados }\end{array}$ & RIBEIRO et al., 2013 & $\begin{array}{c}\text { Estudo observacional, } \\
\text { transversal e } \\
\text { analítico com recurso } \\
\text { a um questionário de } \\
\text { auto resposta, } \\
\text { confidencial e anônimo }\end{array}$ & $\begin{array}{l}\text { Prevalência da disfunção } \\
\text { sexual feminina na idade } \\
\text { reprodutiva }\end{array}$ \\
\hline $\begin{array}{l}\text { A new sexual } \\
\text { revolution }\end{array}$ & ABDO, 2014 & $\begin{array}{l}\text { Análise da quarta } \\
\text { edição do Manual } \\
\text { (DSM-IV), e } \\
\text { comparativo com a } \\
\text { quinta edição desse } \\
\text { manual, DSM-5 }\end{array}$ & $\begin{array}{c}\text { Comparação entre os } \\
\text { Manuais DSM-IV e DSM-V, } \\
\text { onde na atualização a } \\
\text { definição de DGPP passou } \\
\text { para uma só categoria }\end{array}$ \\
\hline $\begin{array}{c}\text { Physical therapy in } \\
\text { sexually } \\
\text { dysfunctional } \\
\text { women: a systematic } \\
\text { review }\end{array}$ & WOLPE et al., 2015 & $\begin{array}{l}\text { Pesquisa descritiva } \\
\text { através da } \\
\text { combinação de } \\
\text { tratamentos } \\
\text { fisioterapêuticos e } \\
\text { disfunções sexuais } \\
\text { femininas }\end{array}$ & $\begin{array}{l}\text { Estudos mostraram melhora } \\
\text { na função sexual após } \\
\text { intervenção fisioterapêutica, } \\
\text { tendo a cinesioterapia } \\
\text { através do TMAP mais } \\
\text { vantajosa e benéfica }\end{array}$ \\
\hline $\begin{array}{c}\text { Autoavaliação vocal } \\
\text { de mulheres na } \\
\text { menopausa }\end{array}$ & BASILIO et al., 2016 & $\begin{array}{l}\text { Pesquisa quantitativa, } \\
\text { transversal, } \\
\text { observacional e } \\
\text { analítica }(n=42) \\
\text { mulheres entre } 19 \text { a } \\
60 \text { anos }\end{array}$ & $\begin{array}{c}\text { Não houve correlação entre } \\
\text { os resultados da } \\
\text { autoavaliação vocal e o } \\
\text { tempo em que as mulheres } \\
\text { pararam de menstruar }\end{array}$ \\
\hline
\end{tabular}


ISSN: 2763-5724

Vol. 01 - n 05 - ano 2021

Editora Acadêmica Periodicojs

\begin{tabular}{|c|c|c|c|}
\hline $\begin{array}{l}\text { Transtornos sexuais } \\
\text { dolorosos femininos }\end{array}$ & BRASIL e ABDO, 2016 & $\begin{array}{l}\text { Classificação dos } \\
\text { TSDs (Transtornos } \\
\text { Sexuais Dolorosos), } \\
\text { etiopatologia e } \\
\text { tratamentos } \\
\end{array}$ & $\begin{array}{l}\text { Os TSDs crônicos são } \\
\text { frequentes, porém muitas } \\
\text { vezes não } \\
\text { diagnosticados }\end{array}$ \\
\hline $\begin{array}{c}\text { Representação } \\
\text { Social das Relações } \\
\text { Sexuais }\end{array}$ & VIEIRA et al., 2016 & $\begin{array}{l}\text { Estudo de campo, } \\
\text { descritivo e qualitativo, } \\
\text { realizado questionário } \\
(\mathrm{n}=60)\end{array}$ & $\begin{array}{l}\text { Informações para a saúde } \\
\text { sexual e o bem-estar } \\
\text { psicossocial das mulheres } \\
\text { através da forma como elas } \\
\text { percebem, sentem e } \\
\text { vivenciam a sua sexualidade }\end{array}$ \\
\hline $\begin{array}{l}\text { Dor na genitália e na } \\
\text { relação sexual }\end{array}$ & FEBRASGO, 2017 & $\begin{array}{c}\text { Conceito sobre DGPP } \\
\text { e vulvodínea e a dor } \\
\text { durante a relação } \\
\text { sexual }\end{array}$ & $\begin{array}{l}\text { Devido à complexidade da } \\
\text { dor, o tratamento da DGPP } \\
\text { requer ações } \\
\text { multidisciplinares }\end{array}$ \\
\hline $\begin{array}{c}\text { Brain Responses to } \\
\text { Vestibular Pain and } \\
\text { Its Anticipation in } \\
\text { Women with Genito- } \\
\text { Pelvic } \\
\text { Pain/Penetration } \\
\text { Disorder }\end{array}$ & PAZMANY et al., 2017 & $\begin{array}{l}\text { Estudo em mulheres } \\
\text { com diagnóstico de } \\
\text { DGPP submetidas à } \\
\text { indução da dor } \\
\text { vestibular no limiar da } \\
\text { dor }\end{array}$ & $\begin{array}{c}\text { Na antecipação e a } \\
\text { indução da dor, as respostas } \\
\text { cerebrais foram mais fortes e } \\
\text { extensas em regiões } \\
\text { envolvidas no processo } \\
\text { cognitivo e aspectos efetivos } \\
\text { da percepção da dor }\end{array}$ \\
\hline $\begin{array}{c}\text { Abordagem } \\
\text { fisioterapêutica da } \\
\text { dispareunia na } \\
\text { mulher com dor } \\
\text { pélvica crônica: } \\
\text { comparação entre as } \\
\text { duas técnicas. Trial } \\
\text { clínico, randomizado }\end{array}$ & SILVA et al., 2017 & $\begin{array}{l}\text { Estudo experimental } \\
\text { em mulheres maior } \\
\text { que } 18 \text { anos, com } \\
\text { dispareunia causada } \\
\text { por espasmos dos } \\
\text { músculos pélvicos e } \\
\text { sexualmente ativas }\end{array}$ & $\begin{array}{l}\text { Tanto a massagem perineal } \\
\text { de Thiele quanto a } \\
\text { eletroestimulação } \\
\text { intravaginal foram efetivas na } \\
\text { melhora da dor e da função } \\
\text { sexual }\end{array}$ \\
\hline $\begin{array}{l}\text { Perineal Massage } \\
\text { Improves the } \\
\text { Dyspareunia Caused } \\
\text { by Tenderness of the } \\
\text { Pelvic Floor Muscles }\end{array}$ & SILVA et al., 2017 & $\begin{array}{l}\text { (n=18)mulheres } \\
\text { separadas em dois } \\
\text { grupos: O grupo } \\
\text { dispareunia }(\mathrm{D}) \text { e o } \\
\text { grupo (DPC) associada } \\
\text { a dor pélvica crônica }\end{array}$ & $\begin{array}{c}\text { Melhora significativa da } \\
\text { dispareunia em ambos os } \\
\text { grupos }\end{array}$ \\
\hline $\begin{array}{l}\text { Physiotherapy for } \\
\text { Pelvic Pain and } \\
\text { Female Sexual } \\
\text { Dysfunction: An } \\
\text { Untapped Resource }\end{array}$ & BERGHMANS, 2018 & $\begin{array}{l}\text { Pesquisas manuais e } \\
\text { assistidas por } \\
\text { computador, } \\
\text { relacionadas à } \\
\text { avaliação } \\
\text { fisioterapêutica e } \\
\text { tratamento da DDGP } \\
\text { e/ou disfunção sexual } \\
\text { feminina }\end{array}$ & $\begin{array}{c}\text { Eficácia e segurança da } \\
\text { fisioterapia pélvica através } \\
\text { de pesquisas clínicas, } \\
\text { contribuindo } \\
\text { significativamente na } \\
\text { avaliação e tratamento da } \\
\text { disfunção }\end{array}$ \\
\hline $\begin{array}{l}\text { Pelvic Floor Physical } \\
\text { Therapy and } \\
\text { Women's Health } \\
\text { Promotion }\end{array}$ & $\begin{array}{c}\text { LAWSON e SACKS } \\
2018\end{array}$ & $\begin{array}{l}\text { Conceitos da atuação } \\
\text { do fisioterapeuta nas } \\
\text { disfunções do } \\
\text { assoalho pélvico }\end{array}$ & $\begin{array}{c}\text { As intervenções } \\
\text { fisioterapêuticas são } \\
\text { variadas e de acordo com o } \\
\text { diagnóstico para melhor } \\
\text { eficácia }\end{array}$ \\
\hline
\end{tabular}

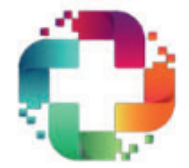


ISSN: 2763-5724

Vol. 01 - n 05 - ano 2021

Editora Acadêmica Periodicojs

\begin{tabular}{|c|c|c|c|}
\hline $\begin{array}{l}\text { Abordagem Da Dor } \\
\text { Gênito- } \\
\text { Pélvica/Penetração }\end{array}$ & TRONCON, 2018 & $\begin{array}{c}\text { Pesquisa bibliográfica, } \\
\text { verificando a } \\
\text { prevalência de dor, } \\
\text { dificuldade persistente } \\
\text { ou recorrente durante } \\
\text { o coito e penetração } \\
\text { vaginal }\end{array}$ & $\begin{array}{l}\text { O artigo visa criar um } \\
\text { protocolo de abordagem da } \\
\text { disfunção, facilitando seu } \\
\text { diagnóstico e tratamento }\end{array}$ \\
\hline $\begin{array}{c}\text { Pelvic floor } \\
\text { rehabilitation in the } \\
\text { treatment of women } \\
\text { with dyspareunia: a } \\
\text { randomized } \\
\text { controlled clinical } \\
\text { trial }\end{array}$ & GHADERI et al., 2019 & $\begin{array}{l}\text { Pesquisa de campo } \\
\text { com objetivo de avaliar } \\
\text { os efeitos das técnicas } \\
\text { de reabilitação do } \\
\text { assoalho pélvico na } \\
\text { dispareunia }(n=84)\end{array}$ & $\begin{array}{l}\text { O estudo mostrou que o } \\
\text { programa melhorou a } \\
\text { disfunção, a função sexual, a } \\
\text { força da MAP e a resistência }\end{array}$ \\
\hline
\end{tabular}

Fonte: Construído pelos autores a partir de PEDro, PubMed e Scielo (2020)

Durante as análises dos artigos, foi possível observar que as DSFs tendem a aparecer na fase fértil feminina, causando dores e desconfortos. Sabemos também que o transtorno da dor gênito-pélvica/penetração (DGPP) engloba junto a ela o vaginismo, dispareunia e a vulvodinia (ABDO, 2014; FEBRASGO, 2017).

Essas informações são necessárias, uma vez que, a prevalência dessas disfunções tem aumentado nos últimos anos, afetando mais de $50 \%$ das mulheres ao redor do mundo. Os sintomas apresentados são: dificuldade à penetração vaginal, medo associado à penetração vaginal, tensão da musculatura do assoalho pélvico à tentativa de penetração. Lembrando que os maiores fatores da dor gênito pélvica (DGPP) estão associados a fatores psíquicos e biológicos, deixando evidente que essas mulheres são afetadas tanto fisicamente quanto mentalmente (RIBEIRO et al., 2013).

Nas revisões abordadas nesse estudo, observa-se que a DGPP é uma síndrome complexa, onde a sensação e intensidade da dor são sentidas em diversos locais. Com isso torna-se difícil o

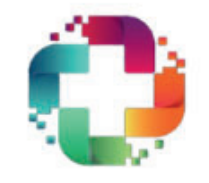


Vol. 01 - n 05 - ano 2021

Editora Acadêmica Periodicojs

diagnóstico, tanto pela vergonha ou bloqueio da mulher em relatar uma queixa sexual, quanto pela falta de perguntas diretas do médico (BRASIL e ABDO, 2016; AMERICAN PSYCHIATRIC ASSOCIATION, 2013).

Em relação à terapia manual no tratamento das DSFs, estudos apontam a eficácia, não só por ter um custo-benefício melhor, mais também por promover a diminuição do tempo de tratamento (SILVA et al., 2017). Segundo o estudo de (WOLPE et al., 2015) a utilização da terapia manual no tratamento de aderências causadas por intercorrências ginecológicas que poderiam provocar dispareunia ou alguma disfunção orgásmica também se mostrou vantajosa pois verificou-se diminuição da dor, melhora das fases do orgasmo onde atua no relaxamento da musculatura pélvica, melhorando o recruta- mento dessa musculatura e aumentando a vascularização local. Assim como (SCAFURI et al., 2009) a massagem perineal ou massagem intravaginal, que é realizada com a mulher em posição ginecológica, tem como objetivo, relaxar a musculatura pélvica, melhorar a circulação sanguínea local e desativa pontos gatilhos.

É feita uma avaliação fisioterapêutica a fim de verificar o tônus e a função dos músculos do assoalho pélvico, a existência e localização de pontos dolorosos, bem como a compreensão de contração e repouso voluntário dos músculos do assoalho pélvico pela mulher. $\mathrm{O}$ tratamento fisioterapêutico tende restabelecer a função dos músculos do assoalho pélvico com uso de intervenções específicas que constam de técnicas de auto relaxamento e controle da ansiedade, melhorando a consciência corporal e utilizando 
ISSN: 2763-5724

Vol. 01 - n 05 - ano 2021

Editora Acadêmica Periodicojs

técnicas de controle respiratório (TRONCON, 2018).

É importante destacar que a conduta te $\neg$ rapêutica relacionada à desordem de dor gê $\neg$ nitopélvica/penetração associados ao ponto gatilho deve ser individualiza $\neg$ da, pois depende das condições clínicas, dos achados da avaliação, bem como dos fatores que predispõem à dor, sendo ele a sensibilidade, impossibilitando assim a realização da massagem intravaginal (FAGE$\mathrm{EH}, 2011)$.

Em relação à vulvodínia, medidas de higiene e cuidado genital adequado utilizando sabão onde o pH ideal é entre 3,5 e 5,5, uso de roupas íntimas de algodão, afastar irritantes vulvares como desodorantes e cremes perfumados, que geralmente contêm álcool, são indispensáveis. Lubrificantes à base de propilenoglicol devem ser substituídos por glicerina ou óleos vegetais. $\mathrm{O}$ uso de anestésicos tópicos como a lidocaína mostrou-se benéfico para a dessensibilização dolorosa vulvar (HAEFNER et al., 2005), per $\neg$ mitindo em alguns casos o retorno à relação sexual, embora não seja indicada como tera-pia de longo prazo (GOLDSTEIN et al., 2016).

Bradley, Rawlins e Brinker (2017) fizeram uma revisão que aborda as técnicas fisioterapêuticas usadas para o tratamento da dor nos músculos do assoalho pélvico, em mulheres com DSFs, considerando a fisioterapia parte integrante da equipe de especialistas para restaurar a função do sistema músculo esquelético em pacientes, assim como Rosembar (2005).

Os objetivos da fisioterapia no tratamento das disfunções do assoalho pélvico com foco nas desordens dolorosas 
são: Aumentar a conscientização e propriocepção da musculatura, melhorar o relaxamento muscular, normalizar o tônus muscular, aumentar a elasticidade da abertura vaginal, dessensibilizar áreas dolorosas e diminuir o medo da penetração, dessa forma a fisioterapia é considerada um importante recurso terapêutico (SILVA, 2017).

Ao analisar todos os estudos podemos verificar que não somente exercícios manuais como exercícios orientados a serem feitos em casa, foram eficazes para a melhora de cada paciente, exercícios esses, que buscam o fortalecimento da MAPs, fortalecendo as fibras musculares lentas e rápidas (KEGEL, 1948; MORKVED et al., 2003). Acreditamos assim que o conhecimento do próprio corpo somado com boa técnica de tratamento fisioterapêutico colabora para me- lhores resultados.

A DSF é um problema que afeta a qualidade de vida de muitas mulheres. O fisioterapeuta sendo parte integrante da equipe multiprofissional pode ajudar consideravelmente na abordagem de avaliação e tratamento dessas mulheres, onde pesquisas científicas comprovam sua eficiência, seus benefícios e segurança.

A utilização da terapia manual mostrou-se eficaz e vantajosa no tratamento das DSFs, sendo de baixo custo, fácil aprendizado e aplicação tanto no atendimento clínico quanto domiciliar e por proporcionar resultados positivos em curto período.

Os resultados do artigo evidenciam que há eficácia em explorar o autoconhecimento para melhora da qualidade de vida das mulheres que sofrem deste problema, proporcionando resultados positivos em um curto 
ISSN: 2763-5724

Vol. 01 - n 05 - ano 2021

Editora Acadêmica Periodicojs

período, contribuindo então para a melhorar da função sexual de mulheres com DSFs. A contribuição do estudo para o campo da Saúde está na necessidade de se pensar políticas públicas que ofereçam às mulheres tratamento adequado para o tratamento das disfunções sexuais femininas. Portanto, mais estudos e pesquisas são necessários para melhorar e contribuir em um diagnóstico mais eficiente, a fim de proporcionar um tratamento mais rápido e eficaz de acordo com as queixas e histórico das pacientes.

\section{REFERÊNCIAS:}

\section{AMERICAN PSYCHIATRIC} ASSOCIATION. Diagnostic and Statistical Manual of Mental Disorders (DSM-5).5th ed. Washington, DC: APA; 2013.

BASILIO, Bianca Natani; RIBEIRO, Vanessa Veis; PEREIRA, Eliane Cristina; et al. Autoavaliação vocal de mulheres na menopausa. Revista CEFAC, v. 18, n. 3, p. 649-656, 2016. Disponível em: $<$ https://www.scielo.br/scielo. php?Pi18462016000300649\&script $=$ sci_abstract\&tlng $=p t>$. Acesso em: 09 de Maio. 2020.

BERGHMANS, Bary. PhysiotheABDO, Carmita Helena Najjar. A new sexual revolution. Einstein, v. 12,n. 2, p. 11-12, 2014. Disponível em: $<$ https://www.scielo.br/scielo.php?Script=sci_arttext\&pi$\mathrm{d}=\mathrm{S} 1679-45082014000200011>$. Acesso em: 15 de Maio. 2020. rapy for pelvic pain and female sexual dysfunction: an untapped resource. International Urogynecology Journal, v. 29, n. 5, p. 631-638, 2018. Disponível em: $<$ https://pubmed.ncbi.nlm.nih. gov/29318334/>. Acesso em: 05 
ISSN: 2763-5724

Vol. 01 - n 05 - ano 2021

Editora Acadêmica Periodicojs

de Maio. 2020.

CREFITO-10: Fisioterapia na

BORNSTEIN, Jacob; GOL- Saúde da Mulher. Crefito10.

DSTEIN, Andrew T.; STOCK- Disponível em: <http://www.

DALE, Colleen K.; et al. 2016 crefito10.org.br/conteudo.jsp?i-

ISSVD, ISSWSH, and IPPS Con- $\quad$ dc=393>. Acesso em: 20 de Maio.

sensus Terminology and Clas- 2020.

sification of Persistent Vulvar

Pain and Vulvodynia. Journal of

FEDERAÇÃO BRASILEIRA

Lower Genital Tract Disease, v.

DAS ASSOCIAÇÕES DE GI20, n. 2, p. 126-130, 2016. Dis-

NECOLOGIA E OBSTETRÍ-

ponível em: <https://3b64welr-

CIA (FEBRASGO). Dor na twev2ibv6q12s4dd-wpengine. genitália e na relação sexual. netdna-ssl.com/wp-content/uploFebrasgo. Disponível em: $<$ htads/2015/09/consensus-terminology-of-Vulvar-Pain-V5.pdf>. Acesso em: 18 de Abril. 2020.

tps://www.febrasgo.org.br/pt/ noticias/item/146-dor-na-genitalia-e-na-relacao-sexual-2017?highlight $=$ WyJ2dWx2b2RcdTAwBRASIL, Ana Patricia Avancini; ZWRuaWEiXQ==>. Acesso em: ABDO, Carmita Helena Najjar. 03 de Junho. 2020.

Transtornos sexuais dolorosos femininos. Revista Diagnóstico GHADERI, Fariba; BASTA\& Tratamento, São Paulo, v. 21, NI, Parvin; HAJEBRAHIMI, n. 2, p. 89-92, 2016. Disponível Sakineh; et al. Pelvic floor rehabiem: < http://www.apm.org.br/ litation in the treatment of women imagens/Pdfs/revista-159.pdf $>$. with dyspareunia: a randomized

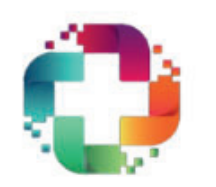


controlled clinical trial. International Urogynecology Journal, v. 30, n. 11, p. 1849-1855, 2019.

Disponível em: <https://pubmed. ncbi.nlm.nih.gov/31286158/>. Acesso em: 28 de Maio. 2020.

HEIM LJ. Evaluation and differential diagnosis of dyspareunia. American family physician, v. 63, n. 8, 2020. Disponível em: $<$ https://pubmed.ncbi.nlm.nih. gov/11327429/>. Acesso em: 29 de Maio. 2020.

LAHAIE, Marie-Andrée; AMSEL, Rhonda; KHALIFÉ, Samir; et al. Can Fear, Pain, and Muscle Tension Discriminate Vaginismus from Dyspareunia/ Provoked Vestibulodynia? Implications for the New DSM-5 Diagnosis of Genito-Pelvic Pain/ Penetration Disorder. Archives of Sexual Behavior, v. 44, n. 6, p. 1537-1550, 2015. Disponível em:
$<$ https://pubmed.ncbi.nlm.nih. gov/25398588/>. Acesso em: 03 de Junho. 2020.

LARA, Lúcia Alves da Silva; SILVA, Ana Carolina Japur de Sá Rosa e; ROMÃO, Adriana Peterson Mariano Salata; et al. Abordagem das disfunções sexuais femininas. Revista Brasileira de Ginecologia e Obstetrícia, v. 30, n. 6, p. 312-321, 2008. Disponível em: <https://www.scielo. br/scielo.php?script=sci_arttext\&pid72032008000600008\&ln$\mathrm{g}=\mathrm{en} \& \mathrm{nrm}=\mathrm{iso} \& \mathrm{tlng}=\mathrm{pt}>$. Acesso em: 18 de Maio. 2020.

LAWSON, Samantha; SACKS, Ashley. Pelvic Floor Physical Therapy and Women's Health Promotion. Journal of Midwifery \& Women's Health, v. 63, n. 4, p. 410-417, 2018. Disponível em: $<$ https://pubmed.ncbi.nlm.nih. gov/29778086/>. Acesso em: 20

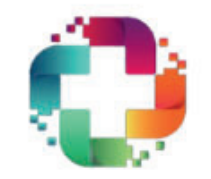


ISSN: $2763-5724$

Vol. 01 - n 05 - ano 2021

Editora Acadêmica Periodicojs

de Junho. 2020.

its anticipation in women with

Genito-Pelvic Pain/Penetration

MENDONÇA, S., \& VOLPON

BERTO, I. (2021). Covid-19

and the increase of the violence

against women in Brazil: over-

coming machine culture. Journal

of Gender and Interdisciplinarity,

2(03). Disponível em https://doi.

org/10.51249/gei02.03.2021.344, acesso em 10/10/2021.

MORRIS, Edward; MUKHOPA-

DHYAY, Sambit. Dyspareunia in gynecological practice. Current

Obstetrics \& Gynecology, v. 16, n. 4, p. 226-233, 2006. Disponível em: $<$ https://www.sciencedirect.com/science/article/ abs/pii/S0957584706000576?via\%3Dihub>. Acesso em: $28 \mathrm{de}$ Maio. 2020.

PAZMANY, Els; LY, Huynh Giao; AERTS, Leen; et al. Brain responses to vestibular pain and
Disorder. NeuroImage: Clinical, v. 16 , p. $477-490,2017$. Disponível em: <https://pubmed.ncbi. nlm.nih.gov/28932680/>. Acesso em: 25 de Maio. 2020.

RIBEIRO, Bárbara; MAGALHÃES, Ana Teresa; MOTA, Ivone. Disfunção sexual feminina em idade reprodutiva - Prevalência e fatores associados. Revista Portuguesa de Clínica Geral, v. 29, n. 1, p. 16-24, 2013. Disponível em: <https://www. rpmgf.pt/ojs/index.php/rpmgf/article/view/11044>. Acesso em: 11 de Maio. 2020.

SILVA, Ana; MONTENEGRO, Mary; GURIAN, Maria; et al. Perineal Massage Improves the Dyspareunia Caused by Tenderness of the Pelvic Floor Muscles. Revista Brasileira de Ginecolo- 
ISSN: 2763-5724

Vol. 01 - n 05 - ano 2021

Editora Acadêmica Periodicojs

gia e Obstetrícia / RBGO Gy-

necology and Obstetrics, v. 39, TRONCON, Júlia Kefalás; PAN-

n. 01, p. 26-30, 2016. Disponível DOCHI, Heliana Aparecida

em: <https://www.scielo.br/scie- da Silva; LARA, Lúcia Alves.

lo.php?script=sci_arttext\&pi- Abordagem da dor gênito-pélvi-

d=S0100-72032017000100026> . ca/penetração. Revista Brasileira

Acesso em: 14 de Abril. $2020 . \quad$ de Sexualidade Humana, v. 28,

n. 2, p. 69-74, 2018. Disponível

SILVA, Ana Paula Moreira da. em: <https://sbrash.emnuvens.

Abordagem fisioterapêutica da com.br/revista_sbrash/article/

dispareunia na mulher com dor view/25>. Acesso em: 15 de Ju-

pélvica crônica: comparação nho. 2020.

entre duas técnicas. Trial clí-

nico, randomizado. 2020. Dis- VIEIRA, Kay Francis Leal; NÓ-

ponível em: <https://teses.usp. BREGA, Renata Pires Mendes

br/teses/disponiveis/17/17145/ da; ARRUDA, Maria Valdê-

tde-19072018-115253/pt-br.php>. nia Soares; et al. Representação

Acesso em: 07 de Abril. $2020 . \quad$ Social das Relações Sexuais:

um Estudo Transgeracional en-

STRAUB, Rainer H. The Com- tre Mulheres. Psicologia: Ci-

plex Role of Estrogens in In- ência e Profissão, v. 36, n. 2, p.

flammation. Endocrine Reviews, 329-340, 2016. Disponível em:

v. 28, n. 5, p. 521-574, 2007 . <https://www.scielo.br/scielo.

Disponível em: <https://pubmed. php?pi98932016000200329\&s-

ncbi.nlm.nih.gov/17640948/>. $\quad$ cript $=$ sci_abstract\&tlng=pt $>$.

Acesso em: 04 de Junho. 2020. Acesso em: 17 de Maio. 2020.

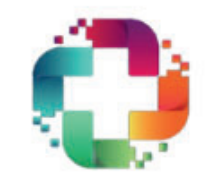

